\title{
Optimizing maternal, newborn, and child health outcomes through use of multidisciplinary 'IMPROVE' teams in Lesotho: Costing component
}

Adebiyi Adesina

Rachael Linder

Lori Bollinger

Follow this and additional works at: https://knowledgecommons.popcouncil.org/departments_sbsr-hiv

Part of the Public Health Commons

How does access to this work benefit you? Let us know!

\section{Recommended Citation}

Adesina, Adebiyi, Rachael Linder, and Lori Bollinger. 2021. "Optimizing maternal, newborn, and child health outcomes through use of multidisciplinary 'IMPROVE' teams in Lesotho: Costing component," Project SOAR Results Brief. Washington, DC: Population Council. 


\section{Optimizing Maternal, Newborn, and Child Health Outcomes Through Use of Multidisciplinary "IMPROVE" Teams in Lesotho: Costing component}

\section{HIV AND PMTCT IN LESOTHO}

Despite its small size, Lesotho has the second highest HIV prevalence globally. HIV prevalence was 23.6 percent in 2018, approximately the same rate which has been observed since $2005 .{ }^{1}$ Women disproportionately share this burden in Lesotho; out of the 330,000 adults living with HIV, 58 percent were women. Additionally, new HIV infections among young women aged 15-24 years were more than double those among young men ${ }^{2}$ and account for approximately one quarter of all new adult HIV infections every year. ${ }^{3}$
Attrition in HIV care and treatment for the general population is around 20 percent; attrition for HIVpositive pregnant women in prevention of motherto-child transmission (PMTCT) programs is similar. ${ }^{4}$ The Lesotho government has attempted to address issues in retention using various interventions; however, the coordination of these at both the facility and community level is somewhat convoluted. For example, there is a lack of standardized job aids or tools to assist in managing patients among cadres at the facility level, to help ensure consistent messaging and care coordination. Furthermore, the role that village health workers (VHWs) play in

\section{KEY FINDINGS}

- The total average unit cost for each of the five services-antenatal care (ANC), family planning (FP), prevention of mother-to-child-transmission (PMTCT), postnatal care (PNC), and pediatric antiretroviral therapy (ART)-was higher in the intervention arm when compared to the control arm of the study. The unit cost for ANC services was \$205 and \$277 in the control versus intervention arms, respectively. The cost of FP was $\$ 81$ in the control arm and $\$ 87$ in the intervention arm. The unit cost for PMTCT services was $\$ 296$ in the control arm versus $\$ 311$ in the intervention arm. The cost for PNC services was $\$ 84$ in the control arm and \$97 in the intervention arm. The cost of pediatric ART was \$321 and \$347 for the control and intervention arms, respectively.

- The cost of the IMPROVE intervention is substantially higher for ANC services (35\%) compared to current treatment protocol in Lesotho. However, the cost for subsequent services were only slightly higher in the intervention arm compared to the control arm-
FP (8\%), PMTCT (5\%), PNC (15\%), and pediatric ART (8\%).

- The cost of the IMPROVE intervention, especially the integrated approach for FP, PMTCT, and pediatric ART, is not prohibitive, and has the potential to improve coordination of care at relatively low cost to existing services.

- Scaling up the intervention would not require a significant amount of financial support or significant human resources.

- As this intervention builds primarily on an already existing healthcare system and infrastructure, there is potential to expand it beyond PMTCT care models.

- The results from this analysis add to the current body of evidence on health costing being collated by the Global Health Cost Consortiuma and can also be utilized for additional analysis (HIV, FP, PMTCT, etc.) and impact modeling applications. 
supporting new mothers in maternal, newborn, and child health (MNCH)/PMTCT care is not clearly linked to facility-based services. While support groups exist for mothers, social and cultural stigma remains and affects uptake of these services.

In response to these challenges, the Elizabeth Glaser Pediatric AIDS Foundation (EGPAF) designed the multidisciplinary "Integrated Management Team to Improve Maternal-Child Outcomes (IMPROVE)" intervention to improve patient-centered care and to boost uptake of family planning (FP) and $\mathrm{MNCH}$ services (specifically, antenatal care [ANC] and postnatal care [PNC]) as well as to improve adherence and retention for patients on antiretroviral therapy (ART). This study builds on the USAID-funded PMTCT service delivery program already being implemented by EGPAF in Lesotho. Rather than designing a suite of entirely new services and delivery platforms, the intervention focused on improving existing services and strengthening the connection between facility and community-based care through:

1. The creation of multidisciplinary integrated management teams, combining facility-based $\mathrm{MNCH}$ providers, VHWs, and representatives from community-based organizations (mothers2mothers [m2m] and Lesotho Network of AIDS Service Organizations [LENASO]), who meet regularly to improve coordination of care and follow-up for patients lost to follow-up.

2. Enhanced Positive Health, Dignity, and Prevention (PHDP)-focused training and job aids to improve care and counseling provided by IMPROVE team members.

3. Increased community-based support via an additional home visit one to two weeks following the first ANC visit to reduce loss to follow-up among new mothers.

Due to various factors, pregnant women have struggled to remain on ART during pregnancy and during the post-partum period. By strengthening team-based care at the facility-level and providing an additional home-based visit during the initial post-partum period, the IMPROVE intervention can help target these points in care where women have historically dropped out. Additionally, it is also expected that there will be broader benefits of the intervention among both HIV-positive and HIV-negative women and their infants, as their care becomes more integrated and actively managed.

From a financial perspective, the cost of implementation is expected to be low, as it leverages the existing health care system and does not introduce any particularly costly components. This brief describes the approach and key quantitative findings of the costing component of the IMPROVE intervention from the service provider perspective.

\section{RESEARCH QUESTIONS}

The aim of the costing component of this study is to determine the cost of the arms-control (current standard treatment protocol) relative to the intervention (IMPROVE)-and to identify key cost drivers for each. Specifically, the aim is to estimate:

1. The cost per person per year (i.e., unit cost) of each of the five key IMPROVE services: general $\mathrm{MNCH}$, which includes both ANC and PNC; FP; PMTCT; and pediatric ART services.

2. The major cost components/drivers of the five key IMPROVE services.

Understanding these costs will provide evidence for those considering the scale up or expansion of the IMPROVE intervention. Evidence from these costing analyses can also help inform policy recommendations to improve existing PMTCT/MNCH services.

\section{METHODS}

IMPROVE used a cluster randomized study design with 12 facilities randomized to receive either routine $\mathrm{MNCH} / \mathrm{PMTCT}$ care based on current treatment protocol plus the IMPROVE intervention, or a control group, which offered routine care based on current treatment protocol only. These facilities were all based in Maseru district, which includes both rural and urban settings, and reflects a range of service delivery modes. A cohort of 1,004 women (614 HIV-negative and 390 HIV-positive) 
were enrolled in the study at their first ANC visit and followed until the last set of study participants recruited were at 12 months post-delivery. The effect of IMPROVE was evaluated via primary outcomes, including treatment retention, adherence, viral suppression, and HIV retesting (perinatally and at 12 and 24 months postpartum), as well as via various secondary outcomes measuring uptake of $\mathrm{MNCH}$ services.

The cost evaluation of facility-based delivery of IMPROVE services began in November 2018. Data collection instruments were designed in collaboration with EGPAF staff to collect data from multiple sources including facility, district, and national health staff and patient forms. After an initial pilot-testing period, adjustments were made to data collection forms to reflect availability of aggregate patient service delivery and cost data. These data were collected from interviews with staff at the facility, district, and national levels of the Ministry of Health and their implementing partners (including EGPAF) by a team of Maseru-based costing consultants. Data on service delivery-types of services offered at the site, number of patients or patient visits by service, and total number of patients or patient visits-were collected from summary registration forms and cross-referenced with MoH's District Health Information Software (DHIS2). The cost data were categorized by the following cost components:

1. Clinical staff costs: These are defined as the estimated time facility-based clinical providers reported spending with the average patient through specific stages (registration, triage, counseling and testing, consultation, adherence counseling, pharmacy, etc.) during a visit by type of service (ANC, FP, PMTCT, PNC, and pediatric ART). The costs are then calculated by multiplying the reported time spent by the average salary of providers by cadre, the average number of staff providing services at each stage of care by staff cadre, and the estimated number of visits per year for each type of service.

2. Laboratory costs: These include the cost of lab commodities (test kits, lab tests, etc.) and the estimated time, reported by lab staff, spent conducting lab tests (blood draw, running test, interpreting test) for each type of patient by service. It also includes the average cost of transporting samples from facilities to testing laboratories (based on national average sample transportation costs). As with clinical staff costs, lab staff costs are then calculated by multiplying the reported time spent running each type of test (HIV, dried-blood spot) by the average salary of staff and the estimated number of tests per patient per year for each type of service. Lab testing occurs at either one of three of the 12 study facilities or at the National Reference Laboratory. Costs were collected from all three facilities, as well as from the National Reference Laboratory. As such, the time spent by lab staff and salary of staff cadres who performed lab services were averaged across these three sources and applied to each of the 12 facilities.

3. Support staff costs: These are defined as time spent by facility-based staff who do not provide direct clinical services to patients but whose work supports these services at the facility (for example, night guard, cleaner, accountant/ bookkeeper). The costs are then calculated for each cadre by multiplying the reported time spent by the average salary of each support staff cadre based at that facility and the estimated proportion of time spent supporting each type of service reported by the same facility. Where the support staff were unable to provide an estimate of the time spent supporting each type of service, costs were estimated by multiplying the staff salary by the proportion of patients or patient visits by service area. The patient proportion by service area is calculated by dividing the number of patients or patient visits for each service area by the total number of patients or patient visits for each facility, respectively, depending on which of these two sets of data is available.

\section{Integrated multidisciplinary management} team costs: This is defined as the average amount of time each facility's multidisciplinary team member spends in meetings to improve coordination of care and follow-up for patients 
lost to follow-up. This cost is calculated by the amount of reported time spent in meetings per month multiplied by the average salary of each team member and the number of meetings a year (12). These costs are apportioned to each of the five service areas for each facility using the number of patients or patient visits divided by the total number of patients or patient visits for each facility, respectively, depending on which of these two sets of data is available.

5. District health management team: This is the cost of the average reported time spent by the Maseru district health management staff supervising and supporting each facility program and the average time spent visiting/supervising each facility per month multiplied by the number of months in a year (12) and the average annual salary of each cadre of the district health management team. These district support teams also include $\mathrm{MOH}$ as well as implementing partner staff.

6. Drugs and commodities costs: This is calculated as the average quantity of each drug, medical commodity, and supply item used during a patient visit for each type of service reported by facility-based clinical providers multiplied by that cost per item of that drug, medical commodity, or supply. The cost of two key drugs and commodities-ARVs and contraceptive commodities ${ }^{\text {a }}$-were calculated using an average weighted cost based on the number of patients on each of the four major ARV regimens used by patients across the 12 sites $^{5}$ and the five contraceptive methods reported in Lesotho's most recent DHS survey, ${ }^{6}$ respectively.

7. Operating costs: This is defined as the cost of utilities (water, telephone, electricity, etc.), transportation (per diem, mobile and emergency vehicle maintenance, etc.), and maintenance costs (building and equipment maintenance). These costs are apportioned to each of the service areas for each facility using the number of patients or patient visits divided by the total number of patients or patient visits for each

${ }^{a}$ National Drug Service Organization-Central Store Price List and UNFPA/Lesotho price list. facility, respectively, depending on which of these two sets of data is available.

8. Equipment costs: These are the cost of medical equipment and furniture estimated by amortizing the cost of each piece of equipment used the year it was purchased, its lifespan/ replacement period, and whether or not it was used for each of the types of services. Like operating costs, these costs are apportioned to each of the service areas for each facility using the number of patients or patient visits divided by the total number of patients or patient visits for each facility, respectively, depending on which of the two sets of data is available.

All financial and cost data were collected in local currency (Maloti) and then converted to U.S. dollars (USD) using the average exchange rate over the study period (November 2017 to October 2019). b All 12 facilities offered ANC, HCT, PMTCT, PNC, and pediatric ART services. Only 5 of the 12 facilities offered FP services-two in the control arm and three in the intervention arm (including one hospital). Some of the costs pertaining to the intervention could not be separated from general program costs, specifically pre-implementation costs for the development of job aids, training at the site level for use of these aids, and start-up costs of the multidisciplinary team meetings, and thus were not available for analysis. The incremental cost of the additional home visit was also not available as it was performed for all patients (including non-study participants) and across all facilities (including nonstudy facilities); as such it could not be allocated to either the control or intervention arm.

To compare between control and intervention facility costs, data were averaged across the six facilities in each of the two arms. Since FP services were only offered in two control and three intervention facilities, FP service costs were averaged for these two groups of facilities for comparison. Lastly, the

\footnotetext{
'International Monetary Fund, Representative Exchange rates accessed via https://www.imf.org/external/np/fin/ert/GUI/ Pages/Report.aspx?CT=\%27ZAF\%27\&EX=REP\&P= DateRange\&Fr $=636051744000000000 \& T o=$ $637079904000000000 \& C F=$ Compressed $\& C U F=$ Period $\&$ $\mathrm{DS}=$ Ascending $\& \mathrm{DT}=\mathrm{Blank}$
} 
percentage cost difference of each service area was calculated by subtracting the total average unit cost of each service in the control arm from the total average unit cost of the corresponding service in the intervention arm, the result of which is then divided by the total average unit cost of that service in the control arm and multiplied by 100 to convert to percent.

\section{RESULTS}

The average cost per person per year for each of the five service areas and the percentage difference (intervention cost relative to control cost) by study arm are shown in Table 1.

As anticipated, the estimated total average unit cost was highest for PMTCT and pediatric ART patients, in both arms. The total average unit cost for PMTCT patients was $\$ 296$ in the control arm versus \$311 in the intervention arm, while the total average unit cost for pediatric patients was \$321 and \$347 for the control and intervention arms, respectively. The total average unit cost for ANC services are \$205 and \$277 in the control versus intervention arms, respectively. However, it is important to note that when the $\$ 14.69$ cost of counseling and testing (including the cost of HIV test kits) are excluded from ANC services, the cost falls to $\$ 190$ and $\$ 263$ in the control and intervention study arms, respectively. The total average unit cost for PNC services is approximately $\$ 84$ in the control arm and $\$ 97$ in the intervention arm. The total average cost per woman per year for FP services in the control arm was $\$ 81$ in the control arm and $\$ 87$ in the intervention arm.

The total average unit cost for each of the services provided in the intervention arm were relatively higher than the total average unit cost in the control arm. The results show that the percentage cost difference for FP, PMTCT, and pediatric ART were 8 percent, 5 percent, and 8 percent, respectively. The intervention cost of PNC was 15 percent higher than the control cost, while ANC had the highest percentage cost difference at 35 percent.

The cost per person by service area is further disaggregated into eight cost categories, which are displayed in Table 2.

Table 1 Cost per woman per year by service area and study arm (USD)

\begin{tabular}{|c|c|c|c|c|}
\hline & $\begin{array}{l}\text { Control } \\
\text { USD }\end{array}$ & $\begin{array}{c}\text { Intervention } \\
\text { USD }\end{array}$ & $\begin{array}{l}\text { Cost difference (intervention } \\
\text { cost-control cost) } \\
\text { USD }\end{array}$ & $\begin{array}{c}\text { Percentage difference of } \\
\text { intervention cost relative } \\
\text { to control cost } \\
\%\end{array}$ \\
\hline Antenatal care & 205 & 277 & 72 & 35 \\
\hline Family planning & 81 & 87 & 6 & 7 \\
\hline РMTCT & 296 & 311 & 15 & 5 \\
\hline Postnatal care & 84 & 97 & 13 & 15 \\
\hline Pediatric ART & 321 & 347 & 26 & 8 \\
\hline
\end{tabular}


Table 2 Unit cost by service and by cost proportions-control and intervention arms

\begin{tabular}{|c|c|c|c|c|}
\hline \multirow{2}{*}{ Service area/cost component } & \multicolumn{2}{|c|}{ Control } & \multicolumn{2}{|c|}{ Intervention } \\
\hline & Cost (USD) & Cost (\%) & Cost (USD) & Cost $(\%)$ \\
\hline \multicolumn{5}{|l|}{ Antenatal care } \\
\hline Clinical staff costs & 46.01 & 22 & 49.19 & 18 \\
\hline Laboratory costs & 108.19 & 53 & 112.39 & 41 \\
\hline Support staff costs & 0.03 & 0 & 0.02 & 0 \\
\hline Multidisciplinary team costs & NA & 0 & 54.78 & 20 \\
\hline District health staff costs & 0.02 & 0 & 0.02 & 0 \\
\hline Drugs and commodities & 8.07 & 4 & 14.63 & 5 \\
\hline Operating costs & 13.39 & 7 & 25.67 & 9 \\
\hline Equipment costs & 29.32 & 14 & 20.51 & 7 \\
\hline \multicolumn{5}{|l|}{ Family planning } \\
\hline Clinical staff costs & 46.37 & 58 & 37.28 & 43 \\
\hline Laboratory costs & - & 0 & - & 0 \\
\hline Support staff costs & 0.03 & 0 & 0.01 & 0 \\
\hline Multidisciplinary team costs & NA & 0 & 22.58 & 26 \\
\hline District health staff costs & - & 0 & 0.02 & 0 \\
\hline Drugs and commodities & 33.82 & 42 & 26.82 & 31 \\
\hline Operating costs & 0.14 & 0 & 0.19 & 0 \\
\hline Equipment costs & 0.26 & 0 & 0.28 & 0 \\
\hline \multicolumn{5}{|l|}{ РМтCT } \\
\hline Clinical staff costs & 89.26 & 30 & 95.66 & 30.8 \\
\hline Laboratory costs & 75.79 & 26 & 80.54 & 25.9 \\
\hline Support staff costs & 0.03 & 0 & 0.01 & 0.0 \\
\hline Multidisciplinary team costs & NA & 0 & 5.28 & 2 \\
\hline District health staff costs & 0.04 & 0 & 0.03 & 0 \\
\hline Drugs and commodities & 117.07 & 39 & 115.84 & 37 \\
\hline Operating costs & 5.33 & 2 & 8.24 & 2.7 \\
\hline Equipment costs & 8.96 & 3 & 4.97 & 1.6 \\
\hline \multicolumn{5}{|l|}{ Postnatal care } \\
\hline Clinical staff costs & 46.01 & 55 & 49.19 & 51 \\
\hline Laboratory costs & - & 0 & - & 0 \\
\hline Support staff costs & 0.02 & 0 & 0.01 & 0 \\
\hline Multidisciplinary team costs & NA & 0 & 10.43 & 11 \\
\hline District health staff costs & 0.02 & 0 & 0.01 & 0 \\
\hline Drugs and commodities & 7.64 & 9 & 10.58 & 11 \\
\hline Operating costs & 13.65 & 16 & 12.22 & 13 \\
\hline Equipment costs & 16.69 & 20 & 14.54 & 15 \\
\hline \multicolumn{5}{|l|}{ Pediatric ART } \\
\hline Clinical staff costs & 124.04 & 39 & 148.72 & 43 \\
\hline Laboratory costs & 76.14 & 24 & 77.09 & 22 \\
\hline Support staff costs & 0.02 & 0 & 0.01 & 0 \\
\hline Multidisciplinary team costs & NA & 0 & 0.41 & 0 \\
\hline District health staff costs & 0.02 & 0 & 0.01 & 0 \\
\hline Drugs and commodities & 119.28 & 37 & 119.28 & 34 \\
\hline Operating costs & 0.38 & 0 & 0.56 & 0 \\
\hline Equipment costs & 0.77 & 0 & 0.51 & 0 \\
\hline
\end{tabular}

6 | Optimizing MNCH outcomes through use of multidisciplinary “IMPROVE” teams in Lesotho: Costing component 
Although the average total cost of each of the service areas was higher in the intervention arm relative to the control arm, when disaggregated into their respective cost components, those differences showed wider variation within each study arm that did not match the same pattern as the total average cost. Additional analysis of the disaggregated cost components showed that these variations were primarily a result of three key input factors. These are:

1. Variation in provider responses on average time spent per patient per service area, the average number of visits per patient per year by service area, the average time spent per patient per visit, and the quantity of drugs and commodities used for each patient per visit. The data collection strategy relied heavily on staff responses, and even though all facilities noted following standard treatment protocols for all five service areas, these treatment protocols do not stipulate standard time spent per patient per visit. Equally important, the quantity of certain drugs and commodities vary by patient need while the number of visits per patient per year are dependent on patient-specific factors (availability, accessibility, and affordability).

2. The allocation key used in apportioning shared costs. The proportion of patient or patient visits by service area assumes that all patients utilize facility resources equally regardless of the type of service provided. In other words, a patient who comes for ANC services receives similar services as a patient who comes for FP, PMTCT, PNC, and pediatric ART. While not the most precise method for apportioning shared costs, this method was the most efficient given the study limitations. Additionally, the method weights $\mathrm{MNCH}$ patients more, particularly ANC patients, who represent the largest share of patients-9 percent of patients in the control arm and 10 percent of patients in the intervention arm.

3. Financial records on operating and equipment costs showed variation within each arm. Hospitals reported higher operating costs, and these costs were higher on average for facilities in the intervention arm relative to the control arm. Similarly, available records showed medical equipment used in the control arm were slightly newer (average purchase date May 2016) than the intervention arm (average purchase date February 2015). This means that on average the estimated value of equipment used for patients in the control arm was slightly higher than in the intervention arm.

With these key variations in mind, the cost drivers are discussed by service area below.

\section{Antenatal care}

Cost drivers for ANC services vary between the control and intervention arms. The largest contributor to costs in the control arm is laboratory services, accounting for 53 percent of the total average ANC cost, a major portion of which is the result of the high cost of HIV, STI, and blood grouping tests. The second largest share of ANC service costs provided under the current guidelines is clinical staff costs (22\%) followed by equipment costs $(14 \%)$, operating costs (7\%), and drugs and commodities (4\%). Similar to control facilities, lab costs account for the largest share (41\%) of ANC services provided by IMPROVE intervention facilities. However, this is followed by the cost of oversight and support provided by the multidisciplinary team (20\%), then clinical staff cost (18\%), operating costs (9\%), equipment costs (7\%), and, lastly, the cost of drugs and commodities (5\%). The key contributing factor to the difference between the study arms is the inclusion of costs associated with the oversight and support provided by the multidisciplinary team at each intervention facility. Of the \$72 (35\%) cost difference between intervention and control facilities, an estimated $\$ 55$ can be attributed to the cost of the multidisciplinary team, representing a substantial two-thirds of the cost difference between intervention and control ANC services.

\section{Family planning}

When disaggregated into the eight cost components, cost drivers for control and intervention facilities mostly follow similar distribution patterns. For FP services in the control arm, costs are split across 
clinical staff costs and the costs of drugs and commodities, with clinical staff costs accounting for the largest share (58\%) while drugs and commodities account for the remainder (42\%). In the intervention arm, FP services are split across three cost components-clinical staff (43\%), drugs and commodities (31\%), and the multidisciplinary team (26\%). In both arms, the allocated costs (support staff, operating costs, equipment costs) account for a minimal share in total costs, less than 1 percent.

\section{PMTCT}

The largest share of the unit cost of PMTCT services is the cost of drugs and commodities, estimated to be 39 percent of the average cost of services at control facilities and 37 percent of the total average cost at intervention facilities, per woman per year. As expected, ARVs account for the largest share of PMTCT costs at 36 percent and 34 percent of the total average unit cost in control and intervention facilities, respectively. After drugs and commodities, the cost of clinical staff time account for approximately 30 percent and 31 percent of total average PMTCT costs in control and intervention facilities, respectively. Laboratory costs account for the third largest share of costs (26\%) of PMTCT services in both the control and intervention facilities. At this point, the cost distribution for both control and interventions diverge. In the control arm, the remainder of the share of cost of PMTCT services are divided into equipment (3\%) and operating costs (2\%). In the intervention arm, the remainder of the share of PMTCT service costs are split by operating (2.6\%), multidisciplinary team (2\%), and equipment $(1.6 \%)$ costs.

\section{Postnatal care}

In control facilities, clinical staff costs account for a little more than half (55\%) of the estimated average unit cost of PNC, followed by equipment costs (20\%), operating costs (16\%), and the cost of drugs and commodities (9\%). In intervention facilities, clinical staff costs also account for a little more than half (51\%) of the share of the cost of PNC services, followed by equipment (15\%) and operating costs (13\%), with the remainder split evenly between the cost of drugs and commodities (11\%) and the multidisciplinary team (11\%). As with ANC cost, the $\$ 11$ cost of the multidisciplinary team represents more than two-thirds of the $\$ 13$ cost difference between PNC services in intervention facilities relative to control facilities.

\section{Pediatric ART}

The cost distribution of pediatric ART services follows the same pattern for both control and intervention facilities. Clinical staff costs represent the largest share of pediatric ART service costs, accounting for 39 percent of total average cost in control facilities and 43 percent in intervention facilities, per child per year. In both study arms, the cost of drugs and commodities account for the second largest share of costs, accounting for 37 percent in control facilities and 35 percent in intervention facilities. Laboratory costs account for the remainder of the share of total average pediatric ART services at 24 percent in control facilities and 22 percent in intervention facilities.

\section{DISCUSSION}

The cost of the IMPROVE intervention, especially the integrated approach for FP, PMTCT, and pediatric ART, is not prohibitive and has the potential to improve coordination of care at relatively low cost to existing services. More specifically, this relatively low-cost difference suggests that the IMPROVE intervention's integrated approach does not require substantial investments to provide integrated services for HIV-positive women and their HIV care. With a relatively low cost, and potential for overall improvement in coordinated care, it is worth exploring the expansion of the IMPROVE intervention beyond PMTCT programming, and to adapt it to additional health areas and care models.

As an entry point for the intervention, the coordinating efforts of the multidisciplinary team adds some costs to ANC services relative to the other service areas. However, this is likely because ANC is often where women needing additional support are identified and providers likely spend more time in the multidisciplinary groups discussing individualized care plans, supplementary support, 
and/or additional home visits The multidisciplinary team's higher cost-share of ANC services relative to the lower cost-share of subsequent services (FP, PNC for HIV-negative women, PMTCT for HIVpositive women), suggests that the early investment in ANC services reduces the need for additional care coordination in PMTCT and other $\mathrm{MNCH}$ services. In an already overburdened and resource strained healthcare system, reducing the need for this additional care management could free up valuable financial and human resources to be utilized elsewhere. The opportunity to repurpose these resources, coupled with the low cost of the intervention, suggests that the IMPROVE model can have multiple beneficial effects upon the overall healthcare system.

A key lesson from the IMPROVE intervention is that there is a need to explore the possibility that integration and coordination across other $\mathrm{MNCH}$ related programs (e.g., malaria, nutrition) at a key service entry point like ANC may reduce the cost of additional support services in the subsequent service areas outlined above (FP, PNC, PMTCT, and pediatric ART). Future studies will need a more comprehensive approach to track patient resourceuse (while ensuring there are ethical safeguards in place) to better understand and document how investment at key service entry points relates to future service utilization and, possibly, gain longterm efficiencies in service delivery.

\section{LIMITATIONS}

- The study was geographically limited to Maseru district, which may not be representative of the entire country.

- Several costs pertaining to the intervention were not available: pre-implementation costs for the development of job aids, training at the site level for use of these aids, and the start-up cost of the multidisciplinary team meetings. The incremental cost of the additional home visit was also not available, as it was performed for all patients (including non-study participants) and across all sites (including non-study facilities); as such it could not be allocated to either the control or intervention arm.
- In most cases, clinical and support staff were only able to provide a best estimate of the amount of time spent with patients in each type of service rather than actual observed time. While it was the most efficient data collection methodology for the study, these estimates are likely subject to reporting bias.

- National averages had to be used as proxy for some cost data (e.g., government lab salaries, laboratory sample transportation). The number of PNC patients or patient visits could not be triangulated with DHIS2 or other sources to verify accuracy. This has implications for the methodology used to apportion costs by each service area's proportion of number of patients or patient clinic visits and overall cost estimates.

- Data for one category of operating costs-external services-were available for only one of the control facilities and were estimated to be over $\$ 20,000$ in one year. The inclusion of these data would have skewed the average operations cost in the control arm, and as such they were excluded from the results.

\section{CONCLUSIONS}

The IMPROVE intervention resulted in a better understanding of inefficiencies at the service delivery level in Lesotho and helped to spark conversations around the importance of patientcentered care and efficient, low-cost methods of improving both facility- and community-based care models. The intervention proved to have a minimal additional cost and is potentially scalable beyond the Maseru district in Lesotho, and beyond PMTCT/ $\mathrm{MNCH}$ services. The collection of cost data also identified areas in which there is limited visibility into cost and operational data (see limitations above), which can potentially help inform the design of future cost studies in similar settings. Cost data from IMPROVE will continue to be utilized beyond this final analysis, and will contribute to a growing database of cost data via the Global Health Costing Consortium, helping to inform future planning methods, models, and analysis focused on HIV testing, PMTCT, MNCH, and FP. 


\section{REFERENCES}

1. Avert. 2019. HIV and AIDS in Lesotho. Retrieved 3 October 2019 from https://www.avert.org/ professionals/hiv-around-world/sub-saharanafrica/lesotho

2. UNAIDS. "AIDSinfo." (Accessed October 2019) https://aidsinfo.unaids.org/

3. UNAIDS. 2018. "Lesotho HIV and health situation room brings innovation to country's AIDS response." Retrieved December 2, 2019, from https://www.unaids.org/en/ resources/presscentre/featurestories/2018/ may/20180509_Lesotho_situation_room.

4. Elizabeth Glaser Pediatric AIDS Foundation (EGPAF), Lesotho country program data. Project SOAR, IMPROVE Protocol.

5. Elizabeth Glaser Pediatric AIDS Foundation (EGPAF), Lesotho country program data.

6. Ministry of Health [Lesotho] and ICF International. 2016. Lesotho Demographic and Health Survey 2014. Maseru, Lesotho: Ministry of Health and ICF International. https://dhsprogram.com/pubs/ pdf/FR309/FR309.pdf

\footnotetext{
Suggested citation: Adesina, Adebiyi, Rachael Linder, and Lori Bollinger. 2021. "Optimizing maternal, newborn, and child health outcomes through use of multidisciplinary 'IMPROVE' teams in Lesotho: Costing component," Project SOAR Results Brief. Washington, DC: Population Council.
}

Project SOAR is a six-year+ (September 2014-January 2021) cooperative agreement funded by the U. S. President's Emergency Plan for AIDS Relief (PEPFAR) and the U. S. Agency for International Development (Agreement No. AID-OAA-A-14-00060). The contents of this brief are the sole responsibility of Project SOAR and Population Council and do not necessarily reflect the views of PEPFAR, USAID, or the United States Government.

Population Council leads the Project SOAR consortium in collaboration with Avenir Health, Elizabeth Glaser Pediatric AIDS Foundation, the Johns Hopkins University, Palladium, and The University of North Carolina at Chapel Hill.
Project SOAR/Population Council 4301 Connecticut Avenue, NW, Suite 280 Washington, DC 20008

Tel: +1202 2379400

e-mail: ProjectSOAR@popcouncil.org projsoar.org

(c) Population Council, January 2021 